\title{
ACUTE UNILATERAL PYELONEPHRITIS, WITH REPORT OF TWO CASES.
}

\author{
By SETON PRINGLE, M.B., F.R.C.S.I.; \\ Surgeon to Mercer's Hospital, Dublin.
}

[Read in the Section of Surgery, April 21, 1911.]

Nowadays the abdomen is so often opened for the relief of acute disease within the peritoneal cavity $I$ think it is of the utmost importance that those extra-abdominal conditions which closely resemble intra-abdominal lesions should receive the attention which will enable us to arrive at correct diagnoses. Most of us, F venture to say, have at one time or another found ourselves in the humiliating position of having operated on a case of supposed acute appendicitis to find a perfectly normal appendix. Therefore, I wish to call your attention to-night to a condition which closely simulates the "acute abdomen," and has frequently been the cause of symptoms which have been put down to acute trouble in connection with the appendix or gall-bladder.

The condition to which I refer is that of acute unilateral infection of the kidney, and I think its importance will be clearly demonstrated by the following report of two cases with which I have had to deal :-

CASE I.-A married woman, aged twenty-four, who was apparently in perfect health except for some frequency of micturition for the preceding week, was seized with acute abdominal pain and vomiting one afternoon at 5 p.m. When the doctor, who referred her to me, was called in the 
same evening at 7 o'clock he found her lying on her back with her legs drawn up. The patient, on being asked to point out the site of the pain, placed her hand so that it lay obliquely between the anterior superior spine of the right ilium and the umbilicus. The right side of the abdomen was rigid and extremely tender to touch both in front and behind in the ilio-costal space. She looked very ill, her tongue was coated, her temperature $103^{\circ}$, and pulse 124 -the whole condition thus closely resembling that seen in acute intraabdominal inflammation. The doctor ordered hot fomentations to be applied, and, as he suspected appendicitis, made arrangements to have her removed to hospital. On her arrival there about 11 p.m. the same night, I was called to see her. Her temperature was then still $103^{\circ}$ and her pulse 126. The very acute pain had, however, passed off, and the tenderness was not marked except in the costovertebral angle behind, but the rigidity of the right abdominal muscles still persisted. Her tongue was dry; she complained of thirst, and was evidently in a state of acute toxæmia. As I suspected the cause of her symptoms, I ordered a catheter to be passed, and the urine thus obtained was examined. It was acid in reaction, the specific gravity was 1026 , it contained a fair quantity of albumen, and there was a heavy deposit of pus, but no red blood cells were dernonstrated by the microscope. The deposit also contained great clumps of a gram-negative bacillus, which I believed to be Bacillus coli communis, and this was afterwards verified by cultivation. I examined her bladder with the cystoscope next morning, and found the base of the bladder congested and the opening of the right ureter inflamed, while the left ureteric orifice was normal. The urine coming from the right side contained flakes of pus, while that obtained from the left by the ureteral catheter was normal except for a few red blood cells. The diagnosis of acute unilateral infection of the right kidney by Bacillus coli communis was therefore established.

Progress and Treatment.- - Her condition remained much the same for eight days. There was moderate right-sided T. 
pain and marked tenderness in the costo-vertebral angle. Her tongue was dry, and thirst was complained of. Her temperature varied from $103^{\circ}$ to $99^{\circ}$, and her pulse-rate from $100^{\circ}$ to $110^{\circ}$. On the third day the rigidity had relaxed somewhat, so that a large, tender kidney could be palpated. On the sixth day I gave her an inoculation of 100 million Bacilli coli communis, the organism having been obtained from her urine, where it occurred in pure culture. On the eighth day I began treatment with anti. colon serum as advised by Dudgeon. I gave the first injection of 15 c.cs. at 6 o'clock that evening. Her temperature was then $102^{\circ}$, and it began to fall rapidly till it reached $97^{\circ}$ ten hours later. It then gradually rose, and was $100.6^{\circ}$ at 6 o'clock the following (ninth) evening, when she received a further injection of 10 c.cs. The temperature again fell to normal and remained so. I should say that I had also given her large doses of sodium bicarbonate with the idea of rendering the urine neutral or alkaline. Following the fall in temperature the patient made a rapid and symptomatically perfect recovery. The urine, however, on her discharge from hospital still contained a few pus cells and bacilli. At present, some six months later, although she has received a course of inoculations with an autogenous vaccine, there are numerous pus cells in her urine, and a pure culture of coli communis can be obtained from it.

CASE II.-A married woman, aged twenty-nine, was sent me by Dr. Powell, of Nenagh, suffering from chronic gastric ulcer. At the operation I found an active pyloric ulcer and also the scar of a healed ulcer, so I performed posterior gastro-jejunostomy. The patient had also a right floating kidney which was particularly easily felt, and during a normal convalescence from the gastric operation, I allowed the students to constantly palpate the organ. On the sixteenth day after the operation I demonstrated this condition to the class, and on that evening she suddenly complained of great pain in the right side of the abdomen and in the back. She vomited and felt shivery, her temperature ran up to $103^{\circ}$, and her pulse to over 100 . On examination there was rigidity and tenderness of the right 
upper abdomen and ilio-costal space, the tenderness being especially marked in costo-vertebral angle. The patient's appearance was toxæmic, her tongue dry, and she complained of thirst. Her urine, which had been normal, now contained albumen, pus, and Bacilli coli. The cystoscope revealed no inflammation of the bladder or orifice of the right ureter, while normal urine was coming from the left ureter, so that the diagnosis of acute pyelonephritis of the right kidney was established. She remained in much the same condition for eight days, the temperature varying from $99^{\circ}$ to $103^{\circ}$, and as I did not at the time know that an anti-colon serum was on the market, and was afraid to use a vaccine in such an acute condition, she was treated by the older methods, and that without avail. Consequently, on the eighth day of her illness, I decided to cut down on the kidney. The kidney was almost twice its normal size, and was intensely congested, being almost black in colour. I therefore split the organ in its entire length along the convex border down into the pelvis. No abscesses were found, so I placed a rubber tube in the pelvis and drew the cut surfaces of the kidney together with a few interrupted stitches, the muscles and skin being also united around the tube brought out on the loin. The result was most satisfactory. Her temperature, which had been $103^{\circ}$ the evening before, was only $97.3^{\circ}$ on the evening of the day on which the operation was performed, and remained normal except for a slight rise a few days later due to some infection around the drainage tube. All her symptoms rapidly cleared up, and convalescence was uninterrupted. The tube was removed on the fifth day, and although urine escaped from the drainage tract for some weeks, this soon ceased, and she went home six weeks after the operation. On her discharge there were still a few pus cells and bacilli to be found in the urine.

The atiology is perhaps the most interesting part in the study of this condition, and has given rise to considerable discussion. On several points all observers are agreed :(1) The great majority of cases occur in females, and 
although pregnancy is a common predisposing cause still the proportion of infection in non-pregnant women far exceeds that in males.

(2) The right kidney is attacked in about 90 per cent. of the cases. We must remember that the right kidney is much more often "floating" than the left, and also that it lies at a lower level, and is thus more liable to injury. In either case the organ is in a state of lowered resistance, and so more prone to infection.

(3) The infecting organism is, in the great majority of cases, the Bacillus coli communis, and it is usually the only organism present. Rarely cases are met with where the offending organism is one or other of the common pyogenic group.

The route by which infection reaches the kidney is, however, the point in the xtiology on which a good deal of controversy has centred. Many observers, such as Barnard, Dudgeon, Bond, and Box, contend that the infection is always an ascending one-that is, that the organism reaches the kidney by means of the ureter travelling up from the lower urinary tract. In support of this theory it is suggested that the preponderance of females in the cases reported is due to the shortness of the female urethra and to the proximity of the external meatus to the anus, from which it may easily be infected by Bacillus coli. Dudgeon has shown that this bacillus is found in the urine in many conditions without producing any symptoms, while Bond has demonstrated the possibility of organisms travelling up mucous passages by means of ascending currents without infecting the tract. These facts would explain not only those cases which follow what may be a mild urethritis or cystitis, but also the cases where no sign of infection of the lower urinary tract could be found on most careful examination. Bond 
further showed that any obstruction to the normal outflow along the canal increases these ascending currents. Such obstructions would occur not only in pregnancy but also in cases of movable kidney causing kinking of the ureter, and in this way the greater frequency of involvement of the right kidney could be explained. Again, the advocates of the ascending infection theory claim that it is difficult to explain unilateral involvement by a blood infection, and also the fact that as a rule no other focus is found in the body.

On the other hand others, among them Brewer and French, claim that the organisms reach the kidney by the blood stream. We know that if any break occurs in the intestinal mucous membrane, the organisms there present can pass in large numbers into the blood, and can be excreted by the healthy kidney without its becoming infected (Metchnikoff, Canon, Buxton and Torrey, Sampson, Brewer). Brewer calls attention to this fact in supporting the hæmatogenous theory of unilateral renal infection, and he describes some experiments which he carried out. He injected cultures of living organisms into the veins of dogs and rabbits, and at the same time injured one of the animal's kidneys, either by bruising it, by injecting bismuth paste into the pelvis, or by ligating one ureter. In the great majority of cases a suppurative lesion was produced in the injured kidney, while the other kidney escaped, and these lesions closely resembled those found at autopsies or operations on cases of acute unilateral pyelonephritis. $\mathrm{He}$, therefore, holds that the Bacilli coli, having gained entrance into the blood stream through a perhaps minute intestinal lesion, are normally excreted by the kidney, but that if, for any reason, the resistance of one or other kidney be lessened at that particular time an acute pyelonephritis results. The condition of lowered resistance may be caused by trauma, by a stone in the 
pelvis of the kidney, or by an obstruction of the ureter, such as may be caused by the pressure of a pregnant uterus or by kinking consequent on floating kidney. By the greater frequency of the latter condition-floating kidney-on the right side, and especially in females, he explains to some extent not only the relative frequency of right-sided infection, but also the fact that the majority of cases occur in women.

There can, I think, be little doubt that the infection may be carried to the kidney by either route, sometimes by the blood stream and sometimes up along the ureter, and I do not think the contention that it invariably reaches the organ in the same way can be upheld. Rolleston and Wright, in dealing with this subject, state their belief that the infection may be either hæmatogenous or urogenous, and the two cases I bring before you to-night are, to my mind, strongly confirmatory of this view. In the first case we have the history of definite bladder irritation preceding the acute symptoms and pointing to primary infection of the lower urinary tract, and this was confirmed by the cystoscopic findings; while in the second case we have the necessary break in the intestinal mucous membrane at the anastomotic opening, and the resistance of the kidney, already low, due to its being "floating," further reduced by excessive handling. We are, therefore, surely entitled to regard the infection in the first as ascending, and in the second as hæmatogenous, of which mode of infection I think the second case furnishes an almost perfect example.

Morbid Anatomy.-The morbid anatomy of the condition has been studied in post-mortem specimens and in kidneys subjected to operation. In the milder forms the organ is enlarged and intensely congested, while subcapsular hæmorrhages are present, microscopically the 
condition is one of "cloudy swelling" and small round cell infiltration. In the severe cases multiple abscesses are found in addition to the enlargement, congestion, and hæmorrhages. Microscopically in some the appearance is one of acute tubular nephritis going on to suppuration (Barnard)-a condition pointing to ascending infection. In others, the abscesses occur in the cortex, and the condition is one of acute suppurative interstitial nephritis, probably due to the plugging of capillaries by masses of organisms (Brewer), and pointing to a blood infection.

Symptoms and Signs.-The symptoms seen in these cases of acute infection show varying grades of severity, as might be expected from a study of the morbid anatomy. A few cases complain of irritability of the bladder for some days preceding the onset, but as a rule apparently the patient has been enjoying perfect health. In all, the onset is acute, with high temperature and rapid pulse. There is usually vomiting and headache, and in some cases rigors occur. The patient looks extremely ill and tox mic. Generally the local signs are marked. There is acute pain in the back and abdomen on the affected side, which is rigid and tender, the tenderness being especially marked in the costo-vertebral angle. The kidney is often felt to be enlarged. In the most acute cases the toxæmia, is very great, and masks the local signs, so that it is very difficult to arrive at a correct diagnosis. In the milder cases the signs pointing to infection of the kidney are generally marked; but here, too, the diagnosis is often by no means easy, and stress must be laid on the tenderness in the costo-vertebral angle. The urine is usually acid, and contains a trace of albumen, pus, a few red blood cells and bacteria, usually a pure culture of Bacillus coli communis. The quantity of pus and blood varies considerably; it has been slight in several reported 
cases and might easily be overlooked, while in others, such as those before you to-night, the deposit was marked. The subsequent progress of the case differs with the degree of severity of the infection. In the most severe or fulminating cases the pain and tenderness continue to be extreme, the toxxmia rapidly increases, and if effective operative interference is not at once carried out the patient quickly succumbs. In the milder cases, in which the toxæmia is not so marked, there is not the same urgency for operation, as undoubtedly the condition has a tendency to undergo spontaneous cure. The toxiemia gradually decreases, the temperature, after being irregular for some days, falls to normal, and the local symptoms subside. Some of the mild cases, however, go on to perinephritic suppuration, and some run a mild chronic course for several months.

Treatment.-In the most severe cases immediate operation is imperative, and nothing less than nephrectomy is of service. Brewer reports thirteen of these cases, five of which were treated by nephrotomy and drainage, and all died; whereas the remaining eight were subjected to nephrectomy, and all recovered. In the milder cases we are justified by the present state of our knowledge to try other methods before proceeding to operation. Urinary antiseptics are of little value, but it is well to render the urine neutral or alkaline by large doses of alkalies. Vaccines of the offending organism have been tried, and, as in most acute infections, are not of much avail; but, on the other hand, Dudgeon reports good results following the use of anti-colon serum in doses of 25 c.cs. spread over three days, and that the serum is worth a trial is evidenced by one of my cases as reported to-night. If, however, in spite of treatment, the toxæmia is not lessening, and the local signs not clearing 
up, the kidney should be cut down upon, and, if not extensively enough involved to necessitate removal, should be split open and drained by a tube brought out into the loin, as was done in my second case with complete success. In the cases which become chronic, vaccine therapy should be persevered with, care being taken to eliminate underlying conditions, such as renal calculus.

In conclusion, might I emphasise the objects which I had in view in bringing this subject before you? They were as follows:-

1. That thorough examination of the urine should be made before operating on cases with signs of acute intraabdominal trouble, more especially on the right side.

2. That in cases presenting acute abdominal symptoms examination should be made for tenderness in the costovertebral angle.

3. That if the abdomen is opened for a supposed acute intra-abdominal lesion, and no such lesion found, the kidney on the affected side should be examined, and if it be found enlarged, or the peritoneum over it congested, a further operation through the loin should be at once undertaken, provided the patient be in a condition to stand the prolongation of the operation.

\section{LITERATURE.}

Barnard. Lancet, 1905. Vol. ПI., pp. 1243.

Bond. Brit. Med. Jour., 1905, Vol. II., pp. 232 ; and Brit. Med. Jour., 1907, Vol. II., pp. 1639.

Box. Lancet, 1908. Vol. I., pp. 77.

Brewer. Surg. Gynæc. and Obstet., May, 1906, and June, 1908.

Buxton and Torrey. Jour. Med. Research, July, 1905.

Cobb. Annals of Surgery, Nov., 1908.

Dudgeon. Lancet, Vol. I., pp. 616 ; and Annals of Surgery, March, 1910.

French. Brit. Med. Jour., 1908. Vol. I., pp. 1033.

Herringham. Clin. Jour., 1910. XXXV., pp. 241.

Sampson. Johns Hopkins Bull. Dec., 1903.

Rolleston. Pract., 1910. LXXXIV., pp. 439.

Wright. Pract., 1909. LXXXII., pp. 344. 
Mr. Gunn said acute coli infection occurred more commonly in children than was generally supposed. Another point of some importance, that while in the majority of cases we get pus in the urine, yet in quite extensive coli infection a clear urine was common. So it is necessary to make a microscopic examination of such cases. $\mathrm{He}$ was called the other day to a lady who was four months pregnant, and had acute pain on the right side of the abdomen, vomiting, and temperature $103^{\circ}$, fast pulse and distended abdomen. The physician in charge thought she had some form of acute obstruction, the surgeon thought it was one of appendix trouble, and as coli was found in the urine, he (the speaker) thought there was a coli infection of the right kidney. On opening the abdomen he found a twisted ovary.

Mr. Stokes raised the question as to whether B. coli might not be present in urine without any inflammation of the kidneys or bladder being present. About four years ago he was requested by the pathologist to examine ten cases of urine from typhoid patients, and coli wère found in them all.

Mr. Pringle said he had not met with cases in children. With regard to the case mentioned by Mr. Gurn he wanted to know was there any tenderness in the costo-vertebral angle behind, as great stress is laid upon it. As regards coli being found in typhoid cases, they are commonly found in urine without there being any symptoms of inflammation. If the kidney has a lowered resistance then an acute infection sets in. 